\title{
Reliable IPTV Service Delivery Using PIM-SSM Routing
}

\author{
A. Quadir ${ }^{1}$, M. T. Arefin ${ }^{2}$, and H. E. Sandström ${ }^{3}$ \\ ${ }^{1}$ Ericsson AB, Färögatan 6, Stockholm, Sweden \\ ${ }^{2}$ Department of CSE, University of Development Alternative, Dhaka, Bangladesh \\ ${ }^{3}$ Mid Sweden University, S 85170 Sundsvall, Sweden
}

Received 9 June 2009, accepted in final revised form 21 August 2009

\begin{abstract}
Internet Protocol Television (IPTV) is a service where digital TV signal data is delivered by using Internet protocol (IP). Telecom and network operators focus on IPTV service for its fastest growth, lower price for operators and market economy. With the improvement of broadband connection and technology, IPTV will get more and more importance. Main challenge for IPTV service is resiliency and Quality of Service (QoS) to meet the users Service Level Agreement (SLA). The design space for IPTV service in terms of resilient backbone design, routing algorithms and fast failure restoration shall be explored. It is described from DSL FORUM and leading IPTV service-providing companies. In the context of this work, the PIM-SSM was configured and QoS was implemented for tagged VLAN of multicast traffic. IPTV traffic is analysed in ACREO testbed and Norrksen network.
\end{abstract}

Keywords: IPTV; QoS; Multicast; PIM; PIM-SM; PIM-SSM; VLAN.

(c) 2009 JSR Publications. ISSN: 2070-0237 (Print); 2070-0245 (Online). All rights reserved.

DOI: $10.3329 /$ jsr.v1i3.2633

J. Sci. Res. 1 (3), 495-507 (2009)

\section{Introduction}

Today's IPTV service is enhancing the consumer's choice, convenience and control of digital entertainment, watching and recording TV programs. Introduction of new services always involves a number of commercial and technical risks. The success of services is dependent on meeting the requirements and expectations of end users. TV channels have been available for a long time over legacy infrastructures including terrestrial broadcast, cable networks and satellite. End users have come to expect a certain level of service regarding availability, latency and quality. Therefore it is vital to keep the technical risks to a minimum and to meet user expectations and needs.

\footnotetext{
${ }^{2}$ Corresponding author: arefin.taslim@cse.uoda.edu.bd
} 


\section{Quality of Service}

IPTV is a real-time service that has very stringent QoS requirements, specifically packet loss, packet reordering, packet duplication, packet faults (bit faults), latency and jitter. A small amount of delay does not directly affect the quality of experience of IPTV. However, a delay longer than 1 second may result a much less-than-satisfying end-user experience. In order to have less than one visible art effect per movie on the TV screen, the packet loss rate must be lower than $10^{-6}$. Fig. 1 provides a snapshot of an IPTV channel with high packet loss. In IPTV service it’s very hard to maintain QoS.

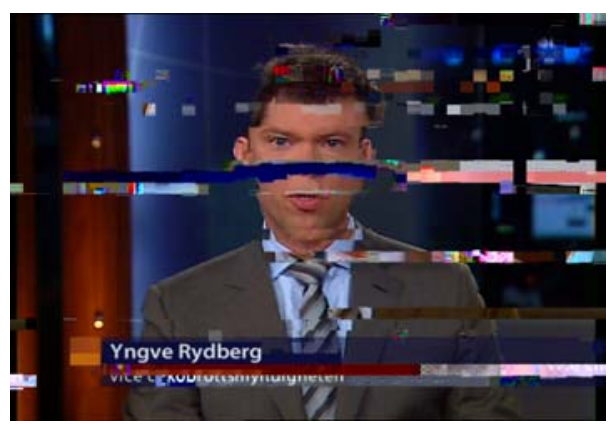

Fig. 1. Effects of packet loss in IPTV service.

\section{Characteristics of IPTV}

IPTV and VoD services need high bandwidth. Depending on the compression and coding technology the following transmission rates should be considered [3]:

a. A MPEG-2 coded SD VoD video stream or IPTV stream is 3.5 to $5 \mathrm{Mbit} / \mathrm{s}$ per TV channel,

b. A MPEG-4 coded SD VoD video stream or IPTV stream is up to $2 \mathrm{Mbit} / \mathrm{s}$ per TV channel,

c. A HD TV channel uses 8 to $12 \mathrm{Mbit} / \mathrm{s}$ when coded with H.264.

The total number of IPTV channels streamed on-line determines the total bandwidth requirements. The total transmission rate of the IPTV content measured in Mbit/s equals the sum of all streams. Each channel is sent only once from the streaming server to the network. The distribution to all subscribers is achieved by multicast implementations in the core and the access networks. Multicast is used to reduce overload. We will discuss in detail about multicast and its QoS.

\section{Multicast Routing}

Multicasting is a technique to distribute datagram to a set of interested receivers on interconnected networks. Challenging network applications such as teleconferencing, Internet Protocol Television, Internet telephony, real-time multimedia gaming, distributed computing, and distributed database applications are growing over Internet. The common point of these applications is that all involve interactions among multiple users forming a 
group and efficient bandwidth with quality of service guaranteed. The need for the multicast applications brings a need for efficient data transfer between many users belonging to the same multicast communication group. Some routing protocols are proposed to meet these conditions. Some of them provide QoS awareness and some of them even provide QoS guarantee.

\section{Protocol Independent Multicast}

In this paper Protocol Independent Multicast (PIM) is described in detail for a better understanding of multicast routing. PIM is widely used multicast routing protocol. Currently, IPTV service network use either PIM-SM or the new protocol PIM-SSM.

\subsection{PIM-SM}

The PIM Sparse-Mode (PIM-SM) protocol is the most widely deployed protocol for IPTV service network. PIM-SM creates a single multicast tree rooted at a core rendezvous point (RP) for all multicast group members within a domain. Sources send their data to the RP which forwards the data down the shared tree to the receivers. A receiver joins a multicast group via IGMPv1 or IGMPv2 and receives data sent by the multicast group source via the shared tree. Distribution via a shared tree can be effective for certain types of traffic, e.g. when the number of sources is large since forwarding on the shared tree is performed via a single multicast forwarding entry. PIM-SM also allows a designated router serving a particular subnet to switch to a source-based shortest path tree for a given source once the source's address is learned from data arriving on the shared tree. For inter domain RP learn about sources in other PIM domains by using the Multicast Source Discovery Protocol (MSDP) [4]. When an active source is identified then RP join that source by shortest path tree and forward data down the local shared tree to the receivers. Currently traditional IP multicast service use IGMPv2 and PIM-SM and MSDP for inter domain multicast. There are some limitations apply with use of IGMPv2, PIM-SM and MSDP [5] like receiver can not specify which specific sources it would like to receive when it joins a multicast group, Address allocation and so on.

\subsection{PIM-SSM}

Source Specific Multicast (SSM) classifies a method of multicast forwarding restricted to shortest path tree to specify sources by hosts, using IGMPv3. The allocation of 232.0.0.0/8 for SSM ensures a range in which SSM is the sole forwarding model. SSM, as implemented by PIM-SM and IGMPv3 requires the following [5]:

a. Source specific host membership report for a particular multicast group. IGMPv3 allows a host to describe specific sources from which it would like to receive data.

b. PIM shortest path forwarding. Source specific host report for a particular multicast group and initiating PIM (S,G) joins directly and immediately as result.

c. Eradication of shared tree forwarding. In order to achieve global effectiveness of SSM, all networks must agree to restrict data forwarding to source trees for some 
recognized group range. The address range 232.0.0.0/8 has been allocated by IANA for use by source specific multicast (SSM).

\subsection{Benefits of PIM-SSM}

The following benefits can be acived by using PIM-SSM [5]:

a. SSM provides a basis for access control mechanisms. A single source $\mathrm{S}$ can transmit to a channel (S,G) where $\mathrm{G}$ is an multicast address. Each receiver is capable of specify the specific sources from which it would like to receive. The elimination of shared tree forwarding prevents consuming network resources based on $(*, G)$ forwarding.

b. SSM defines multicast channels on a per-source basis such that SSM addresses are "local" to each source. This prevents the problem of global allocation of SSM addresses for multicast groups. Each source is now responsible for resolving address collisions for the various channels that it creates.

c. The multicast tree for an SSM channel $(S, G)$ is always rooted at the source S. Thus there is no need for an RP-based shared tree infrastructure or for MSDP for source discovery. That is why complexity of the multicast routing infrastructure for SSM is lower than PIM-SM.

\section{Service Structure for IPTV}

Telecom Operators are facing the challenge of declining voice revenues due to increased competition and the mass proliferation of mobile communications. In addition, Cable TV operators are adding voice services to their traditional cable network and thus entering into the domain of telecommunication carriers. Nowadays triple play service is main focus point for telecom operators, especially IPTV service. IPTV service faces resiliency problem, technical difficulties and challenges. Incomplete service offerings and unexpected quality degradation of the triple play services resulted in negative impact on end users and increased customers. To maintain and establish a stable, QoS-guaranteed network for triple play service, it should follow the standard service architecture that proposed by some big companies like CISCO, SIEMENS, JUNIPER. IPTV service architecture includes Core Network and Access Networks. Basic structures for IPTV services [6] have Super Hub Office (SHO) where IPTV traffic is sourced and Video Hub Office (VHO) where video streams are received from SHO. Two networks should be established for IPTV service. One is the core network and the other one is the access network. SHO situate in the core network and VHO situate in the access network.

\subsection{Service architecture}

The IPTV service architecture [6] includes a core network and an access network. Assume that there are two locations from which IPTV traffic is sourced, known as the Super Hub Office, or SHO. Two SHO provide redundancy to ensure reliable video 
transmission. Video streams transmitted from SHO and received at VHO, where the video streams further processed and then transmitted towards the IPTV customers. VHO also store local video content to support VoD. Each VHO consists of video equipments and routers. For redundancy each VHO should connect to the SHOs. Fig. 2 illustrates the service architecture.

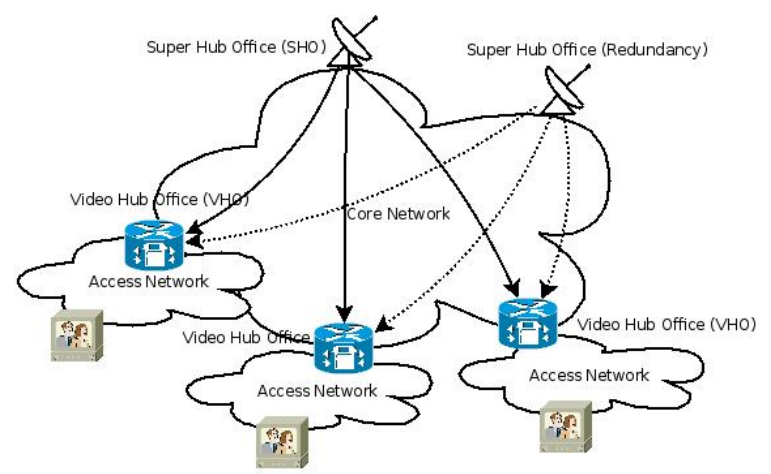

Fig. 2. Service architecture of IPTV.

\subsection{Core network}

The core network is considered as IP network. The core network based IS-IS or OSPF or on an MPLS backbone for underlying unicast routing. These algorithms route traffic over the shortest path between source to destination and based on the administrative weights assigned to each network links. For multicast traffic, we assume that there is a single multicast tree including the two SHOs and all the VHOs. The SHOs are assigned a common anycast source address so that each VHO is routed to the nearest SHO as it joins the multicast tree. Thus, traffic is transmitted from the nearest SHO to each VHO, and each VHO receives a single copy of the multicast traffic at any given point in time. If a link from SHO to VHO goes down then the backup link from SHO will deliver data to that VHO. This creates redundancy of SHO. VHO is automatically re-routed to the SHO if any failure occurs.

\subsection{Access network}

The DSL Forum has recently finished the specification [2,3] for the fundamental architecture for Ethernet based DSL aggregation networks or fiber network, which includes also definitions for the delivery of multicast services such as IPTV services. TR101 and TR-126 differentiate between two different approaches for connecting broadband users to the aggregation network, firstly the so-called 1:1 also known as VLAN per subscriber, and secondly the N:1 also known as VLAN per service mode. VLAN allows 
the network to logically segment the network into multiple communities by "tagging" each packet with a field which identifies packet ownership. VLAN usage is standardized as IEEE 802.1Q, and are sometimes called "VLAN tags" or "Q-tags".

\subsubsection{VLAN per subscriber}

In the VLAN per subscriber model, there is a dedicated VLAN for each subscriber. This approach is called Customer-VLAN (C-VLAN). The C-VLAN is created between the access elements (PON, ONT/OLT, DSLAM) and the Broadband Service Router (BSR) and carries all traffic for all services to and from an individual subscriber. This model mirrors the proven edge architecture used in many carrier environments for dial-up, private line, Metro Ethernet, and Frame/ATM aggregation. Since there is a 1:1 mapping of a C-VLAN to a subscriber, a single physical connection from the DSLAM to the edge router supports 4095 subscribers. In other words, each DSLAM can support 4095 subscribers. The 1:1 mapping of VLAN to customers enables the edge router to effectively manage the bandwidth for each subscriber. The 1:1 approach is shown in Fig. 4.

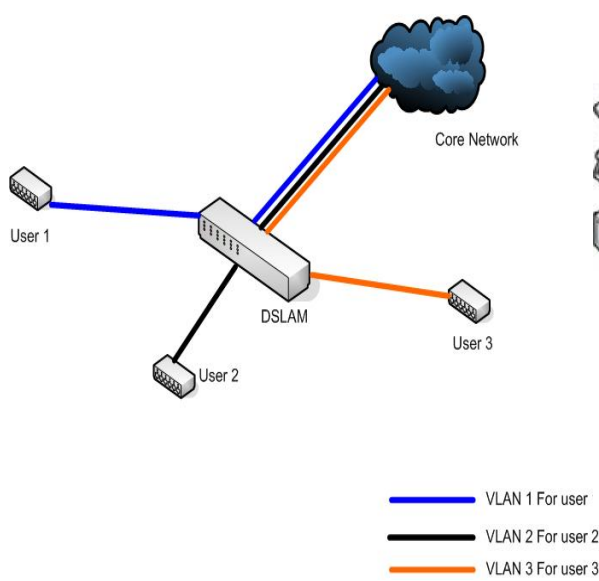

Fig. 4. VLAN for subscriber.

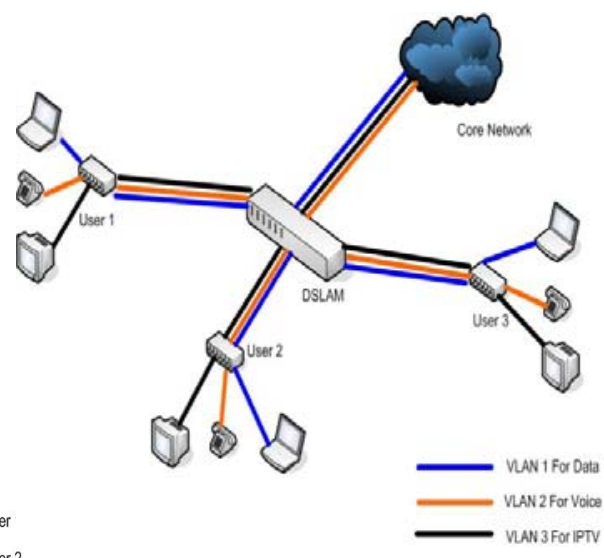

Fig. 5. VLAN per service.

\subsubsection{VLAN per service}

In the Service VLAN model, there is a shared VLAN used to deliver services to subscribers. A separate VLAN is used for each service. This architecture is frequently used when IPTV service is introduced onto a broadband network. Putting new services into a different VLAN lowers the risk of disrupting the existing service. This approach is shown in Fig. 5. 


\section{Performance and Analysis}

One of the most challenging and important service for the development of broadband network and telecom is IPTV. It has great impact both on technical and economical aspects. Quality of Service and resiliency are the main technical focus point for IPTV. In this section some IPTV measurement is discussed. The measurement is done on the ACREO AB TESTBED network. For more research a new Norrsken network is going be established between 14 cities.

Acreo AB [7] has a testbed project gained a wide acceptance and in many cases unique experience on IPTV. This project includes practical broadband access network design, different access technologies and types of equipments, broadcast and transmission of IPTV streams, as well as maintenance and support of IPTV service. This testbed is implemented in Hudiksvall, Sweden. Sweden is among the leading countries worldwide concerning IPTV service. However, only $2 \%$ of the populations are watching IPTV, and most of them are watching IPTV in the municipality network over FTTH.

\section{IPTV infrastructure in ACREO Testbed}

Acreo AB [7] has used the fiber infrastructure of the municipality network in Hudiksvall. Different vendor equipments have been used to establish the testbed network. The broadband access network was designed with layer 2 and layer 3 switches. Triple play service was offered in this testbed network. IPTV was tested in different access technologies:

- Layer 2 based active optical network

- Layer 3 based AON [FTTH solution]

- Ethernet passive optical network, EPON [FTTH solution]

- ADSL2+

A total of around 100 test pilots had access to IPTV during the project period. This test pilot is offered 40-50 channels including several films channels.

\section{IPTV QoS measurement}

An IPTV network is illustrated in Fig. 6. This testbed uses PIM-SM for multicast routing. IPTV traffic is measured in a test apartment. Video quality measurement and multicast traffic testing is done by Agama RIA 2.2 and Multicast ping. Agama Technologies [8] offers end to end monitoring solutions for the IPTV service. The Agama IPTV Monitoring Solution is a complete and distributed real time monitoring tools for IPTV services. It listens to IPTV channels just like a customer set-top box. The incoming channels are analyzed and results are stored into a database. Agama Analyzer detects errors on all protocol layers, both the network and MPEG layer. In the network layer it detects problems as packet loss, jitter and reordering problems. Agama Analyzer contains a complete MPEG decoder and analyzes the selected transport streams. Having a complete analysis of the MPEG protocol stack makes sure that that the Analyzer catches 
errors, regardless if they originate from the network, head-end or are inherent in the MPEG stream. The analysis is not only on the pure technical level. Agama Analyzer categorizes the problems and display how serious the effects are using a heuristics engine. This enables Analyzer to differentiate between a hardly detectable minor distortion and more serious errors. The result of the analysis is presented in a web based user interface. Agama supports the monitoring of multiple multicast channels, depending on channel bit rates and performance of the machine. The Agama Analyzer node is a software solution and can be installed on a standard x86 Linux machine. The user can then choose the most appropriate hardware platform for their specific need. It can be used as a standalone monitor or integrated into an Agama Enterprise Server system. Ssmping [9] is a tool for checking the multicast link for SSM. Another tool called asmping [9] is also provided to check ASM. Using these two tools, multicast traffic is measured from the test apartment.

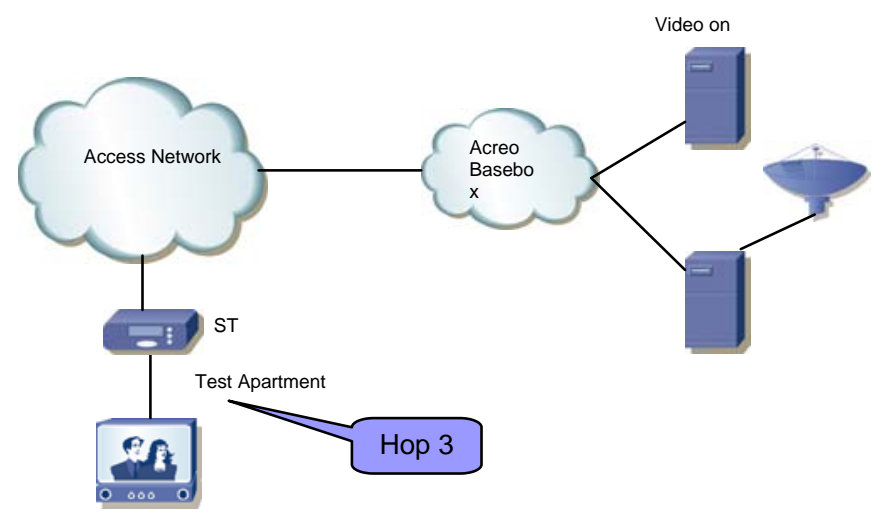

Fig. 6. ACREO IPTV test bed network in Hudiksvall (Sweden).

On this testbed, IPTV quality was measured by Brunnstrom [7] in 2005, with the result 99.5\% IPTV traffic without errors. In 2007 the IPTV traffic found so much error due to proper network structure, proper equipment for IPTV and increased the IPTV traffic after 2 years. Fig. 7 shows the traffic analysis by Agama analyzer. Channel SVT1 is measured here. Fig. 1 shows the channel and its distortion due to network or traffic problems. At that time there is major distortion in SVT1 found by Agama analyzer. By using Agama it's hard to tell where the problem in IPTV traffic in network around in picture between 11:00 to $12: 30$ as well as $13: 30$ to $14: 40$ as well as $15: 15$ to $15: 30$. It is only shows the problem in the traffic. For further investigation asmping and ssmping are used.

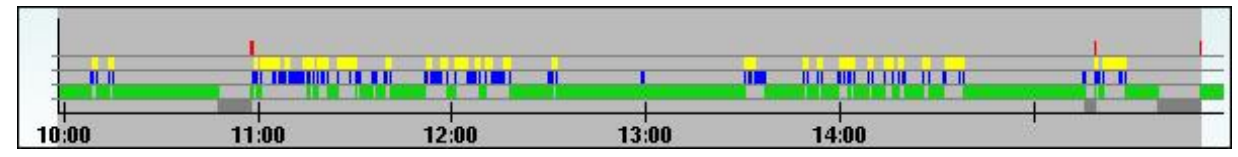

Fig. 7. TV channel measurement graph from Agama. Green=OK, Blue=minor distortion, Yellow=major distortion, Red=Packet loss. 
Multicast ping is a very useful tool to find out the network problems. By using ping it is easy to measure packet losses, time-to-live (ttl) and hop count. Multicast ping and unicast ping is measure from test apartment. In Fig. 8(a), there is statistics how much loss is found from the end user by using the multicast and unicast ping. In the measurement it is found that there is 5 to $6 \%$ loss in both unicast and multicast traffic. That means there are problems in testbed network and no priority is set for multicast traffic. These measurements were done on $26^{\text {th }}$ to $29^{\text {th }}$ July 2007.

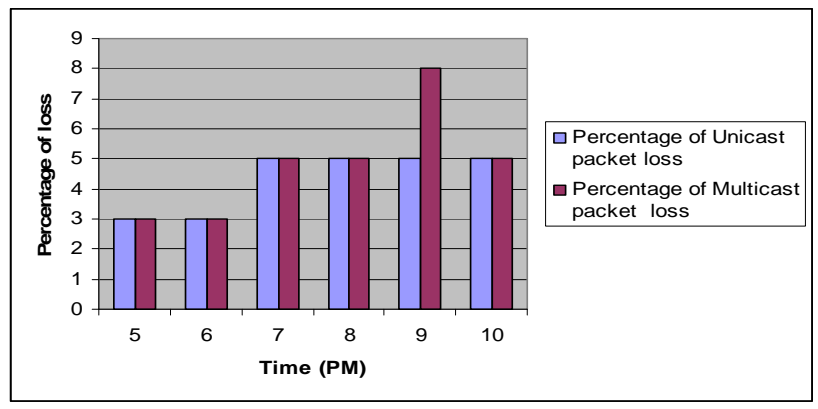

Fig. 8 (a). Packet loss measurement.

In Fig. 8 (b), multicast ping test is done from test apartment at hop 3 and got $10 \%$ loss. 65000 multicast packets were tested to check the multicast links and also unicast links. Then again this test is done in hop 2 and got $0 \%$ loss. Fig. 8(c) shows the result of ping test.
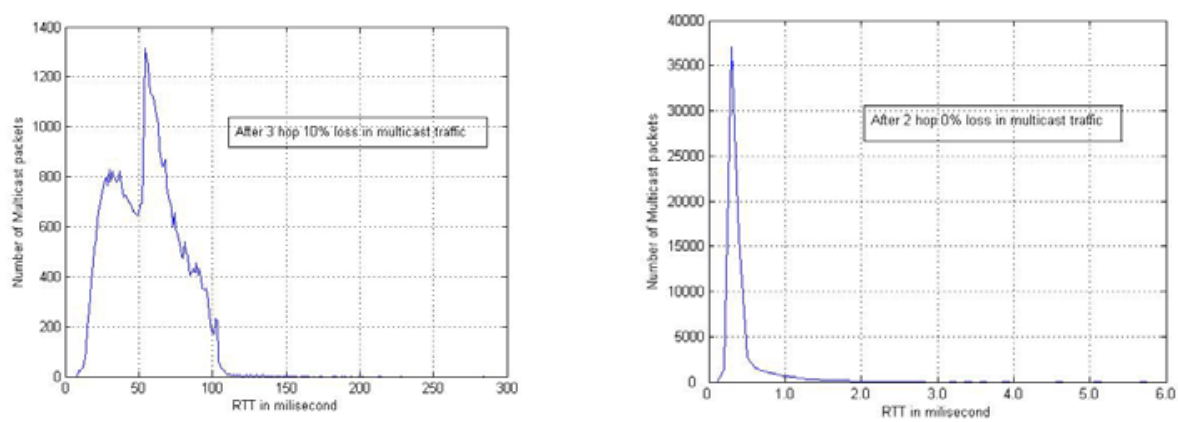

Fig. 8 (b). Multicast packet measurement at hop 3. Fig. 8 (c). Multicast packet measurement at hop 2.

65000 packets transmitted, time $65000001 \mathrm{~ms}$ unicast:

59001 packets received, $9 \%$ packet loss

$\mathrm{rtt} \mathrm{min} / \mathrm{avg} / \mathrm{max} / \mathrm{std}-\mathrm{dev}=0.657 / 5.422 / 1017.162 / 4.727 \mathrm{~ms}$ multicast:

58467 packets received, $10 \%$ packet loss since first mc packet (seq 2) recvd $\mathrm{rtt} \mathrm{min} / \mathrm{avg} / \mathrm{max} / \mathrm{std}-\mathrm{dev}=0.762 / 5.538 / 1017.190 / 4.749 \mathrm{~ms}$ 
After these tests, it is found that some network equipment like switches have some problems. Switches are important in networks and switches may behave differently for different types of traffic. Some switches are tested afterwards. Switches are Cisco 3745, 3COM and Allied tel. These three types of switches are tested for multicast traffic with 80 channels on both channel switching and without channel switching mode. Cisco is better than Allied telsys and Allied telsys is better than 3com for IPTV service. It may happen that these switches have different input and output buffer size, and due to different switch fabrications. After changing some switches and ports the result is found; Fig. 9 continues to depict the graph for the SVT1 channel. Even though it is improved, there is still some distortion in the channel.

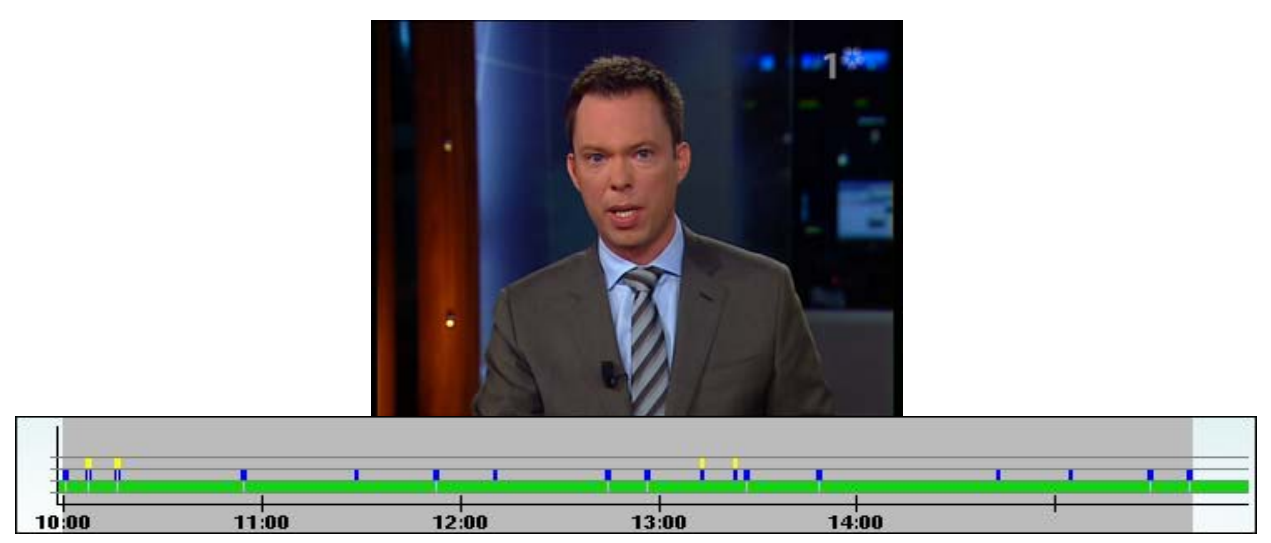

Fig. 9 TV channel measurement graph from Agama. Green=OK, Blue=minor distortion, Yellow=major distortion, Red=Packet loss.

Research Network for IPTV

It is very hard to find out the traffic problem in IPTV service. Many factors can influence the degradation of QoS for IPTV traffic. Both active and passive measurements should be done for figuring out the factor of traffic degradation. The purpose of this Norrsken research network is to measure a number of quality indicators. The measurement nodes are normal PC's with dual Ethernet ports. One port is used for IPTV traffic measurements and this port can be conFig.d to listen to multiple 802.1q tagged networks. All fault conditions will be analyzed, and how to improve resilience is the main focus of this research project. Causes for degradation of quality are bit faults on transmission links, faults in active equipment (switches/routers), congestion, and configuration errors.

The capacity of the network nodes and the total network load also set the conditions where the network can operate in a non-congested state. Faults that occur in congested situations must be handled differently than faults in non-congested situations. Another purpose of the measurements is to determine how often these congestion situations occur 
and if congestion situations are local or isolated or occur at multiple places at the same time, possibly interdependent. In a congested situation the individual network component (switch/router) still have a few choices;

a. It can drop incoming packets instead of putting them on an outgoing queue;

b. It can take a late decision and drop an outgoing packet and thus transmitting the next packet from an outgoing queue instead of the first;

c. It can choose other queuing schemes than first in first out for priority packets;

d. If there are some feedback facilities in place it can inform the upstream router/switch about the congested condition

Since routers or switches operate with highly specialized circuits, there are limits on how flexible the above features are on a given network component. So any given congestion scheme has to work on a large enough subset of network components to be useable.

A multimedia stream is composed of multiple layers of information. Some of this information can be dropped with only minor quality consequences. These packets can be marked as low priority. Some of the information is crucial to maintain the stream integrity. These packets can be marked as high priority. And even more important is the fact that there are some packets that are necessary for the control of the network integrity, like re-routing messages. These are even higher priority. Sadly, the current way of transmitting real-time video streams encapsulated in the so called Transmission Stream (TS) format does not make this kind of TS to network packet mapping possible. If it was possible a diffserv scheme with 64 levels of priority would probably be very useful information for the network components when dealing with congested situations. However this level of granularity is not handled by most switched/routers that currently only can handle 2, 4 or 8 priority queues/levels.

\section{Research Network Setup}

Fig. 6 shows the network architecture for research IPTV network. The main IPTV source is in Hudiksvall, Sweden. Here the ACREO IPTV source is used and distributed over the network. CISCO 3745 router is used for connect with ACREO IPTV network. The research network is configured for PIM-SSM and service VLAN. Links to other cities are connected by Norrsken fiber link and use CWDM technology for separate channel for research network. For multicast traffic measurement dbeacon is used. Dbeacon [10] stands for Distributed Beacon. Dbeacon is a Multicast Beacon written in $\mathrm{C}++$. The main purpose of a beacon is to monitor and collect statistics such as loss, delay and jitter between beacons. Dbeacon support both IPv4 and IPv6 multicast, collecting information using both Any Source Multicast (ASM) and Source-Specific Multicast (SSM). By using dbeacon we gather the information from the Sundsvall to Hudiksvall link. Fig. 10, Fig. 11 and Fig. 12 show the multicast traffic loss, delay and jitters between Sundsvall to Hudiksvall link. We found loss when we flood the link from Hudiksvall to Sundsvall. When congestion occurs in the link both delay and jitter increase, and packet losses 
appear. On $25^{\text {th }}$ September the link from Hudiksvall to Sundsvall found around 5\% loss in the link, delay increased to $14 \mathrm{~ms}$ and jitters also increased around $10 \mathrm{~ms}$. There are some gaps in the graphs due to service unavailability because of setup change and upgrade.

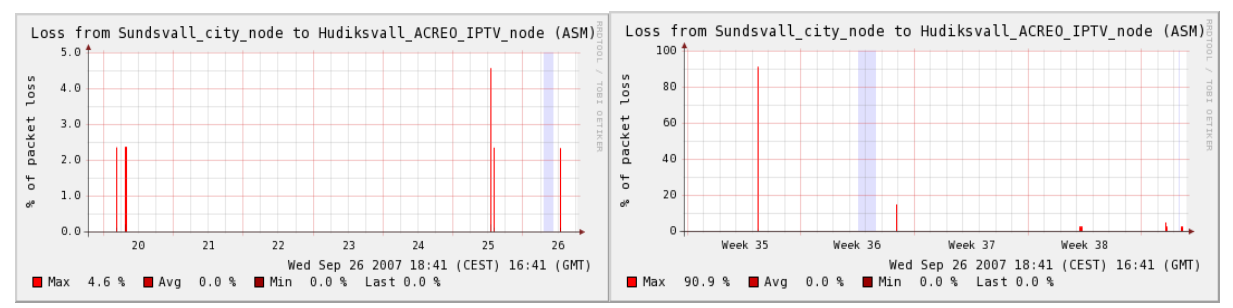

Fig. 10. Packet loss in multicast beacon program.

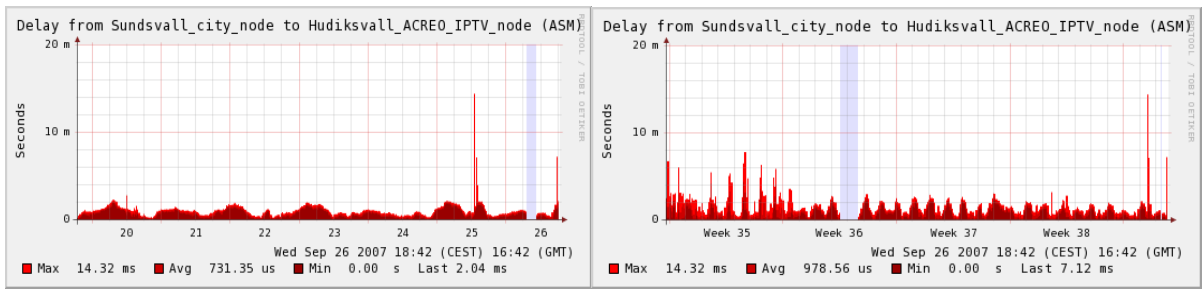

Fig. 11. Delay in multicast beacon program.

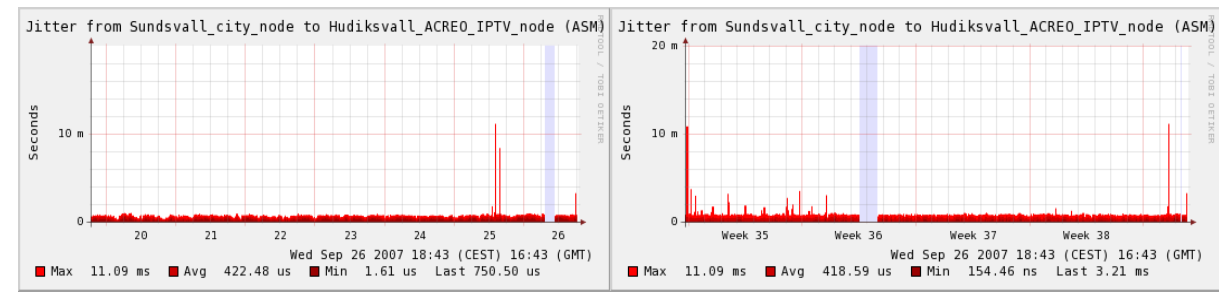

Fig. 12. Jitters in multicast beacon program.

In this context of this work, the PIM-SSM was configured and QoS was implemented for tagged VLAN of multicast traffic.

\section{Conclusions}

On the background that user expectations of high quality IPTV service deliver over broadband network is a main challenge for telecom and Internet service providers and this continuous service availability and QoS should not be compromised even if a failure occurs in network, this work addresses resilient service architecture. In this work, IPTV traffic in the Acreo testbed and research network was measured and analyzed. We found network architecture and QoS configuration problems for multicast traffic in Acreo testbed. Additionally, we implemented PIM-SSM, tagged VLAN for multicast traffic and provided QoS settings for the research network. According to these results and observations some suggestions are given here for resilient IPTV service. 
a. For Multicast, use PIM-SSM for source specification features,

b. Use service VLAN to separate the multicast traffic,

c. Use a proper network structure for resilient IPTV service,

d. Active equipment (switches/routers) should be configured properly.

It is very difficult to measure and predict the problem of multicast traffic in a network. Here active measurement is done. Passive measurement gives more detailed information. So there is the need for passive measurement of multicast traffic for getting detailed QoS factors in live network. Further works are continuing. Our work was done with IPv4 network. There are interesting scopes of research work for IPTV traffic measurements on IPv6 network.

\section{References}

1. Kerpez, D. Waring, G. Lapiotis, J. Lyles, and R. Vaidyanathan, IEEE Communications 44 (9), 166 (2006). doi:10.1109/MCOM.2006.1705994

2. T. Rahrer, DSL Forum Technical Report TR-126 - Triple Play services Quality of Experience (QoE) Requirements (December 2006).

3. M. Cha, G. C, J. Yates, A. Shaikh, and S. Moon. Case study: and M. Resilient backbone design for IPTV services. IPTV Workshop, International World Wide Web Conference, Edinburgh, Scotland, United Kingdom (May 23, 2006).

4. D. Estrin, L. Wei, D. Farinacci, A. Helmy, D. Thaler, S. Deering, M. Handley, V. Jacobson, C. Liu, and P. Sharma, Protocol independent multicast sparse mode (PIM-SM): Protocol specification, Internet Engineering Task Force (IETF), RFC 2362 (June 1998).

5. S. Bhattacharyya, A Framework for Source-Specific IP Multicast Deployment - Internet Draft, draft-bhattach-pim-ssm-00.txt (July 2000).

6. Siemens Communications and Juniper Networks, I., High Quality and Resilient IPTV Multicast Architecture (2006).

7. A. Loa, A. Roaf, and A. Gunnar, Acreo Broadband Communication Project Report Y2002Y2006 (January 2007) Acreo AB.

8. Agama Technology (cited 2007 2nd June; End-to-End IPTV Monitoring Solutions). Available at: http://agama.tv/index.html

9. SSMPing. (cited 2009 15th May); ssmping is a tool for checking whether one can receive SSM from a given host]. Available at: http://www.venaas.no/multicast/ssmping/.

10. dbeacon (cited 2009 16th May); dbeacon is a Multicast Beacon written in C++. The main purpose of a beacon is to monitor other beacon's reachability and collect statistics such as loss, delay and jitter between beacons. dbeacon support both IPv4 and IPv6 multicast, collecting information using both Any Source Multicast (ASM) and Source-Specific Multicast (SSM).]. Available at: http://fivebits.net/proj/dbeacon/. 